\title{
Cristal adaptatif refroidi pour rayonnement $X$
}

\author{
D. Dézoret, R. Marmoret, A. Freund* et R. Ravelet**
}

CEA, Service Conception Expérimentale des Mesures, BP. 12, 91680 Bruyères-le-Châtel, France

* European Synchrotron Radiation Facility, BP. 220, 38043 Grenoble cedex, France

** Laserdot/Groupe Aérospatiale, 91460 Marcoussis, France

\begin{abstract}
Résumé : Nous présentons l'étude d'un cristal adaptatif destiné à équiper une ligne de lumière d'un synchrotron de type E.S.R.F. Ce cristal est constitué d'un monocristal de silicium de faible épaisseur fixé sur un support refroidi en béryllium. Un système d'actionneurs piézo-électriques permet de corriger les déformations du cristal produites par le rayonnement synchrotron. La première étude, thermomécanique, montre que la pente au niveau de la face avant du cristal n'excède pas $3 \mu \mathrm{rad}$ dans le sens de propagation des rayons $X$. La seconde étude, portant sur l'assemblage silicium/béryllium, nous oriente vers la méthode d'assemblage par adhérence moléculaire.
\end{abstract}

\section{INTRODUCTION}

Avec le développement des synchrotrons de troisième génération, les larges domaines spectraux et les brillances élevées qui les caractérisent nécessitent des appareillages optiques adaptés. En effet, les fortes puissances et les densités élevées de photons produites par ce type de source y créent des dépôts d'énergie élevés $(1,2,3)$.

Dans le cas d'un monochromateur à cristaux, les gradients de température engendrent une courbure générale et une déformation locale auxquelles peut s'ajouter une augmentation de la distance entre plans atomiques. Par conséquent, les performances du monochromateur (divergence angulaire du faisceau monochromatisé, résolution en énergie et intensité transmise) sont affectées. Dans le cas où nous utilisons un monocristal de silicium de faible épaisseur, la courbure générale est la composante principale des déformations.

Pour minimiser ces déformations, plusieurs solutions ont déjà été étudiées ${ }^{(4,5)}$. Le principe de l'optique adaptative utilisée en astronomie a été demièrement étendu au domaine des rayons $X$ grâce à la réalisation d'un miroir asservi( $(6,7)$. Nous étudions la transposition de ce principe au premier cristal d'un monochromateur à deux cristaux. Après une description du cristal et de son dispositif d'asservissement, nous présentons les résultats de l'étude thermomécanique et des tests des assemblages silicium/béryllium( ${ }^{(8)}$. 


\section{CARACTERISTIQUES DES SOURCES DE LUMIERE}

Ce cristal a été optimisé pour deux types de ligne de lumière de l'E.S.R.F. $(9,10,11)$. La première source est un aimant de courbure et la seconde est le wiggler ID11 (BL2, Material Science). Leurs caractéristiques principales sont réunies dans le tableau 1.

Sur la ligne de lumière $\mathrm{n}^{\circ} 2$, nous avons tenu compte du miroir adaptatif, utilisé en amont du cristal, avec une orientation telle que son énergie de coupure soit à $20 \%$ au dessus de l'énergie de travail đu cristal. Le tableau 2 donne, pour le domaine d'énergie $5-60 \mathrm{keV}$, la puissance totale et la densité de flux thermique au niveau du cristal.

\begin{tabular}{|c|c|c|}
\cline { 2 - 3 } \multicolumn{1}{c|}{} & Aimant de courbure & Wiggler ID11 \\
\hline Ec & $19 \mathrm{keV}$ & $28,8 \mathrm{keV}$ \\
\hline $\mathbf{P}$ & $0,42 \mathrm{~kW}$ & $5,3 \mathrm{~kW}$ \\
\hline $\mathbf{P}_{\mathbf{0}}$ & $<1 \mathrm{~W} / \mathrm{mm}^{2}$ & $<30 \mathrm{~W} / \mathrm{mm}^{2}$ \\
\hline$\sigma_{\mathbf{v}}$ & $0,1 \mathrm{mrad}$ & $0,1 \mathrm{mrad}$ \\
\hline$\sigma_{\mathrm{h}}$ & $6 \mathrm{mrad}$ & $2 \mathrm{mrad}$ \\
\hline
\end{tabular}

Tableau 1 : Caractéristiques des sources de lumière (énergie des électrons $6 \mathrm{GeV}$, courant $100 \mathrm{~mA}$ ) Ec énergie critique, $P$ puissance totale, $P_{0}$ densité de flux thermique à $30 \mathrm{~m}$ de la source, $\sigma \mathrm{v}$ divergence verticale à l'énergie critique (FWHM), oh divergence horizontale (FWHM)

\begin{tabular}{|c|c|c|}
\cline { 2 - 3 } \multicolumn{1}{c|}{} & Aimant de courbure & Wiggler ID11* \\
\hline $\mathbf{P}(\mathbf{k W})$ & 0,42 & $0,4<\mathbf{P}<3,9$ \\
\hline $\mathbf{P o}\left(\mathbf{W} / \mathbf{m m}^{\mathbf{2}}\right)$ & $0,02<\mathbf{P o}_{0}<0,23$ & $\mathbf{0}, 9<\mathbf{P o}<1,3$ \\
\hline
\end{tabular}

Tableau 2 : P puissance totale et Po densité de flux thermique à la surface d'un cristal de Si(111) placé à $30 \mathrm{~m}$ de la source *l'énergie de coupure du miroir adaptatif est placée à $20 \%$ au dessus de l'énergie de travail du cristal

\section{DESCRIPTION DU CRISTAL ADAPTATIF ET DE SON DISPOSITIF D'ASSERVISSEMENT}

\subsection{Description du cristal}

Le cristal adaptatif est représenté sur la figure 1. I se compose d'un monocristal de silicium fixé sur un support refroidi en béryllium et d'une embase piézo-électrique. Les dimensions totales de la pièce sont $120 \mathrm{~mm}$ par $90 \mathrm{~mm}$ sur $110 \mathrm{~mm}$ de haut. La zone active a une épaisseur de $2,3 \mathrm{~mm}$ sur $70 \mathrm{~mm}$ par $70 \mathrm{~mm}$. La pièce est refroidie à l'eau par 35 canaux de $1,3 \mathrm{~mm}$ de diamètre sur $120 \mathrm{~mm}$ de long. Deux canaux de distribution et de collecte sont usinés perpendiculairement aux 35 canaux. Pour transmettre leur mouvement au cristal, les actionneurs piézo-électriques sont fixés sur des plots de $5,5 \mathrm{~mm}$ de diamètre usinés directement dans le support en béryllium. Le sommet des plots est détouré sur $1 \mathrm{~mm}$ de hauteur pour former des pions de $1 \mathrm{~mm}$ de diamètre. Ces pions font office de rotule. Afin d'éviter tout collage ou assemblage, le support en béryllium(12) est 
usiné dans un seul bloc. Un écran en dénal (alliage de tungstène) protège les actionneurs piézo-électriques des rayons $\mathrm{X}$.
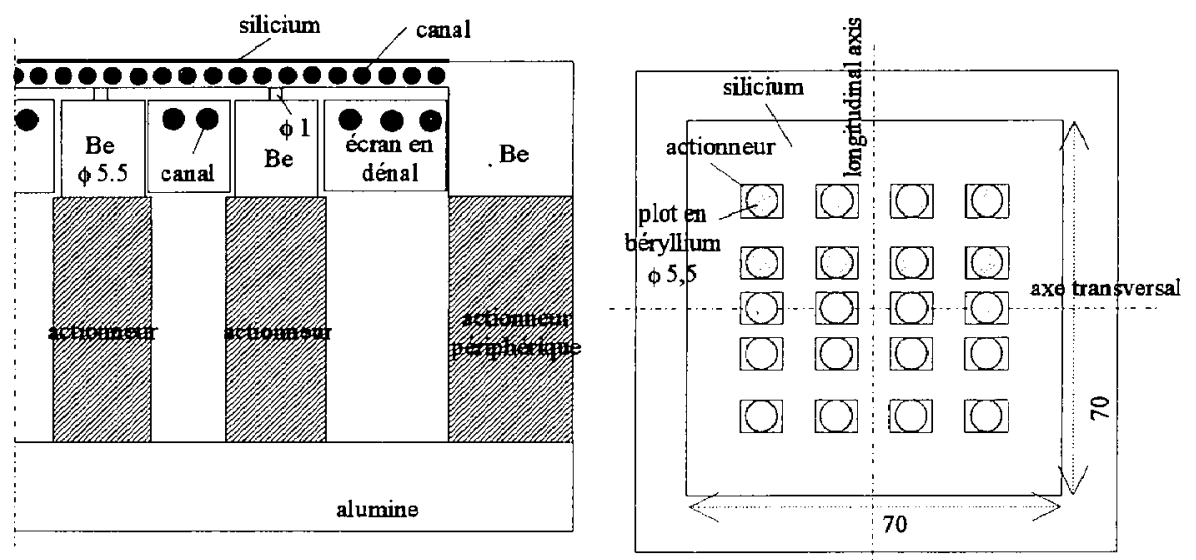

Figure 1 : schéma du cristal adaptatif (coupe transversale et disposition des actionneurs)

\subsection{Description du système adaptatif}

Une optique adaptative(13) se compose d'un miroir déformable, d'un analyseur de front d'onde et d'un calculateur de commande (figures 2 et 3). Dans cette étude, le support en béryllium et le monocristal font office de miroir. Les actionneurs piézo-électriques (20 actionneurs ponctuels et 1 actionneur périphérique) modifient la forme du cristal.

Une source de lumière éclaire la surface du cristal. Sous l'effet de la charge thermique due au rayonnement synchrotron, la surface déformée du cristal modifie le front d'onde. Un dispositif de micro-lentilles placé à l'entrée d'une CCD reforme autant d'images de la source sur la rétine de la caméra qu'il y a de micro-lentilles. Le déplacement des images, $y$, est une fonction de $\alpha$, pente locale, et de $f$, distance focale des micro-lentilles. Une relation matricielle relie le déplacement des images de la source aux tensions appliquées aux actionneurs et par conséquent à la forme du cristal.

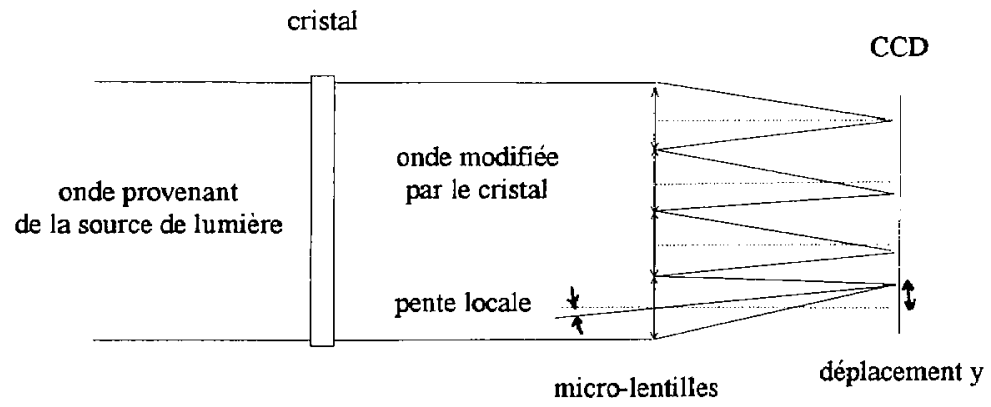

Figure 2a : Principe du cristal adaptatif 


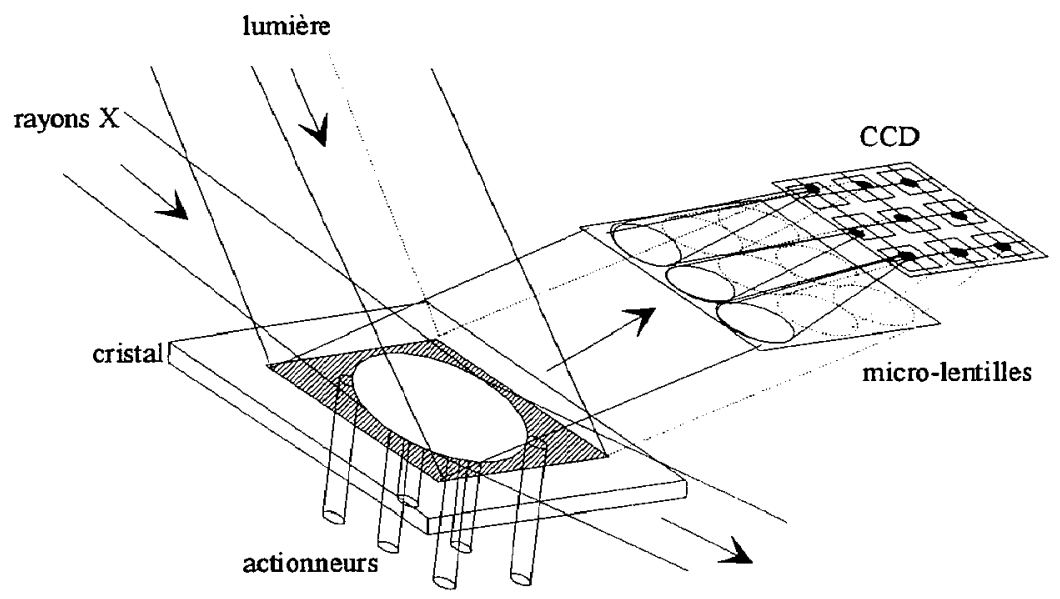

Figure 2b : Principe du cristal adaptatif

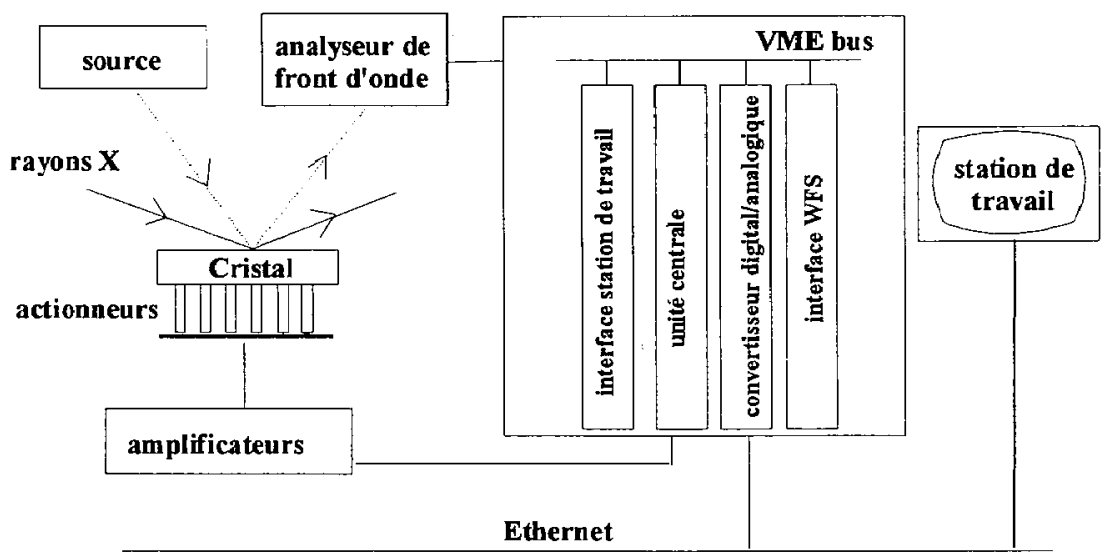

Figure 3 : Schéma général du dispositif d'asservissement

\section{SIMULATION}

\subsection{Choix des matériaux}

Le but de cette étude est d'obtenir un cristal dont la surface a une pente inférieure à $5 \mu \mathrm{rad}$ dans le sens de propagation des rayons X. Cette valeur de $5 \mu \mathrm{rad}$ correspond à un élargissement de $10 \%$ de la largeur de Darwin du silicium (111) à $25 \mathrm{keV}$ environ.

Afin de déterminer la distribution de température, les déformations du cristal et la forme du système adaptatif refroidi, une étude thermomécanique a été effectuée à l'aide du code aux éléments finis I-DEAS.

La charge thermique induite par le rayonnement synchrotron provoque une courbure générale de l'optique ainsi qu'une déformation locale. La pente résiduelle associée à ces déformations est une fonction de la densité de flux thermique absorbée et du facteur $\alpha / \kappa$, 
où $\alpha$ représente le coefficient d'expansion thermique et $\kappa$ la conductivité. En tenant compte de l'absorption sélective des rayons $X$ par les matériaux, nous avons simulé un cristal mince fixé sur un support transparent au rayonnement $X$. Le béryllium a été choisi à cause de sa faible absorption des rayons $X$ dans le domaine $5-60 \mathrm{keV}$. Le facteur $\alpha / \mathrm{k} \mathrm{du}$ béryllium est 2,5 fois plus grand que celui du silicium (tableau 3 ) mais son absorption est 6 fois plus faible.

\begin{tabular}{|c|c|c|c|}
\cline { 2 - 4 } \multicolumn{1}{c|}{} & $\boldsymbol{\alpha}\left(\mathbf{K}^{-\mathbf{1}}\right)$ & $\mathbf{K}(\mathbf{W} / \mathbf{c m} . \mathbf{K})$ & $\boldsymbol{\alpha} / \mathbf{K}(\mathbf{c m} / \mathbf{W})$ \\
\hline Silicium & $2,3610^{-6}$ & 1,50 & $1,5710^{-6}$ \\
\hline Béryllium & $7,7010^{-6}$ & 1,93 & $3,9910^{-6}$ \\
\hline
\end{tabular}

Tableau 3: Caractéristiques du silicium et du béryllium à $273^{\circ} \mathrm{C}$

\subsection{Première simulation : comparaison entre un système silicium et un système silicium/béryllium}

La première simulation est une comparaison entre un système tout en silicium et un système composé d'un cristal mince de silicium monté sur un support en béryllium. Dans les deux cas, le système est refroidi par de leau circulant dans des canaux et asservi par 16 actionneurs piézo-électriques ponctuels fixés en face arrière du système. La densité de flux thermique est de $1 \mathrm{~W} / \mathrm{mm}^{2}$ et l'angle d'incidence est de $90^{\circ}$. Dans les deux cas, la puissance absorbée dans le silicium est simulée par une absorption en surface tandis que celle absorbée dans le béryllium est simulée par un dépôt volumique. Le coefficient d'échange de l'eau a été fixé à $20000 \mathrm{~W} / \mathrm{m}^{2 \circ} \mathrm{K}$ et la vitesse de l'eau dans les canaux à $4 \mathrm{~m} / \mathrm{s}$.

Les résultats de la simulation sont reportés dans le tableau 4. Les pentes maximales sont données dans les cas non asservis et asservis. Les résultats suivant l'axe de propagation des rayons $\mathrm{X}$ montrent que la pente maximale du système silicium/béryllium est inférieure d'un facteur 8 à celle du système tout silicium.

\begin{tabular}{|c|c|c|c|c|}
\cline { 2 - 5 } \multicolumn{1}{c|}{} & \multicolumn{2}{c|}{ silicium } & \multicolumn{2}{c|}{ silicium/béryllium } \\
\cline { 2 - 5 } \multicolumn{1}{c|}{} & passif & asservi & passif & asservi \\
\hline$\Delta \mathbf{z}^{\prime}(\mu \mathrm{rad})$ & 60 & 7 & 35 & 5 \\
\hline$\Delta \mathbf{x}^{\prime}(\boldsymbol{\mu r a d})$ & 60 & 36 & 40 & 4,5 \\
\hline$\Delta \mathbf{T}$ & \multicolumn{2}{|c|}{$27^{\circ}$} & \multicolumn{2}{c|}{$4^{\circ}$} \\
\hline
\end{tabular}

Tableau 4: Résultats des systèmes silicium et silicium/béryllium (1 ère simulation) $\Delta \mathrm{T}$ : différence entre la température de la face avant du centre du cristal et la température ambiante, $\Delta z^{\prime}$ : pente suivant l'axe de propagation des rayons $X, \Delta x^{\prime}$ : pente suivant l'axe transversal à la direction de propagation des rayons $\mathrm{X}$

\subsection{Seconde simulation : système silicium/béryllium en géométrie de Bragg}

Ce second calcul a permis de simuler le système silicium/béryllium en géométrie de Bragg et d'optimiser le nombre et la position des actionneurs piézo-électriques. La densité de flux thermique est $1 \mathrm{~W} / \mathrm{mm}^{2}$ et les énergies de travail sont $5 \mathrm{keV}$ et $23 \mathrm{keV}$ (tableau 5). La puissance absorbée par le silicium est simulée par un dépôt en surface et celle absorbée par le béryllium par un dépôt volumique. Nous avons tenu compte de l'absorption par 
effet photoélectrique des rayons $\mathrm{X}$ par les matériaux. Nous avons négligé l'effet Compton. La distribution gaussienne de la densité de flux thermique $P\left(d, x^{\prime}, z^{\prime}\right)$ dans le cristal est de la forme :

$$
P\left(d, x^{\prime}, z^{\prime}\right)=P_{0}(d) \exp \left(-x^{\prime 2} / 2 \sigma_{x}^{2}\right) \cdot \exp \left(-z^{\prime 2} \sin ^{2} \theta / 2 \sigma_{z}^{2}\right)
$$

où $P o(d)$, densité de flux thermique, décroît de manière exponentielle avec la profondeur $\mathrm{d}$ des matériaux. $\sigma \mathrm{x}$ et $\sigma z$ sont les écarts-types des dimensions du faisceau de rayons $\mathrm{X}$ et $\theta$ est l'angle entre la direction du faisceau et la surface du cristal.

Nous avons simulé un cristal de silicium (111) de $10 \mu \mathrm{m}$ d'épaisseur fixé sur le support en béryllium de 2,3 $\mathrm{mm}$ d'épaisseur refroidi à l'eau. Les actionneurs ponctuels sont au nombre de 20 et un actionneur périphérique a été ajouté.

Le tableau 5 donne la valeur des pentes maximales. Les résultats sont meilleurs dans cette seconde simulation et sont inférieurs à la limite souhaitée suivant la direction de propagation des rayons $X$.

\begin{tabular}{|c|c|c|}
\cline { 2 - 3 } \multicolumn{1}{c|}{} & $\mathbf{5 ~ k e V}$ & $\mathbf{2 3} \mathbf{~ k e V}$ \\
\hline Puissance incidente & $420 \mathrm{~W}$ & $960 \mathrm{~W}$ \\
\hline Puissance absorbée dans le silicium & $24 \mathrm{~W}$ & $90 \mathrm{~W}$ \\
\hline Puissance absorbée dans le béryllium & $76 \mathrm{~W}$ & $90 \mathrm{~W}$ \\
\hline $\boldsymbol{\Delta z}^{\prime}(\boldsymbol{\mu r a d})$ & 3 & 3 \\
\hline $\boldsymbol{\Delta} \mathbf{x}^{\prime}(\boldsymbol{\mu r a d})$ & 8 & 27 \\
\hline $\boldsymbol{\Delta} \mathbf{T}$ & $4^{\circ}$ & $5^{\circ}$ \\
\hline
\end{tabular}

Tableau 5 : Résultats du système silicium/béryllium ( $2^{\text {nd }}$ simulation)

\section{ASSEMBLAGE SILICIUM/BERYLLIUM}

Sur le support en béryllium, nous souhaitons fixer un monocristal de silicium de dimensions utiles $70 \mathrm{~mm}$ par $70 \mathrm{~mm}$. L'épaisseur optimale serait, une fois le cristal poli, de $20 \mu \mathrm{m}$. Plusieurs types d'assemblages peuvent être réalisés. Si un matériau intermédiaire au silicium et au béryllium doit être utilisé, il ne doit pas contribuer à l'échauffement et à la déformation du cristal. Ce matériau doit donc absorber faiblement les rayons $\mathrm{X}$, être de faible épaisseur et bon conducteur de la chaleur.

Nous avons réalisé trois types d'assemblages : le collage à l'aide de colle céramique, la soudure par diffusion d'aluminium et l'adhérence moléculaire. Pour chacun des assemblages, un cristal épais de silicium (111) est fixé sur une plaque de béryllium. Le cristal est progressivement aminci et, entre chaque amincissement, nous traçons sa courbe de diffraction (diffractomètre 3 axes E.S.R.F.). La réflectivité d'un cristal parfait de $\mathrm{Si}(111)$ est de $90 \%$ environ et la largeur à mi-hauteur de sa courbe de diffraction est de 6 arcsec à $8 \mathrm{keV}$ (raie $\mathrm{K} \alpha$ du cuivre).

La colle céramique a donné de bons résultats (figure 4) mais elle n'a pas tenu aux rayons $\mathrm{X}$. Les résultats obtenus par soudure à l'aluminium se dégradaient au cours de l'amincissement. En effet, au fur et à mesure que l'épaisseur de silicium diminuait, les contraintes mécaniques dues à l'assemblage apparaissaient. L'adhérence moléculaire est la solution que nous avons retenue. L'épaisseur finale obtenue est pour l'instant $100 \mu \mathrm{m}$. 
Difirion dahxriiun

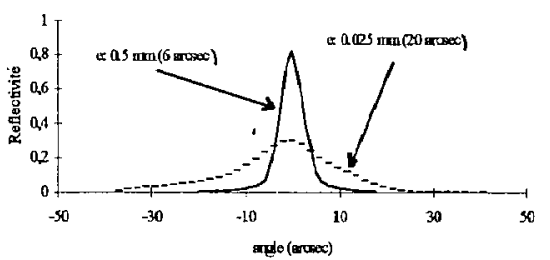

Adhérence meléalare

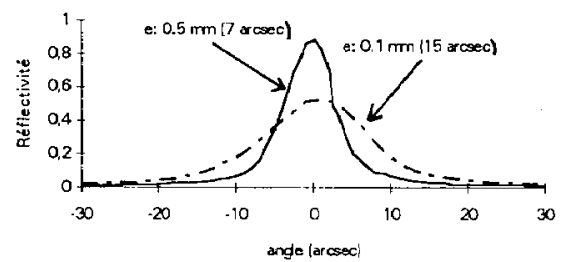

Dffesiond'duminium

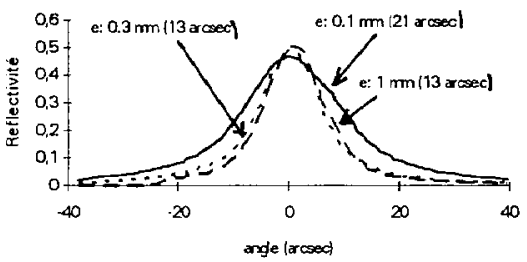

Colle cíatique

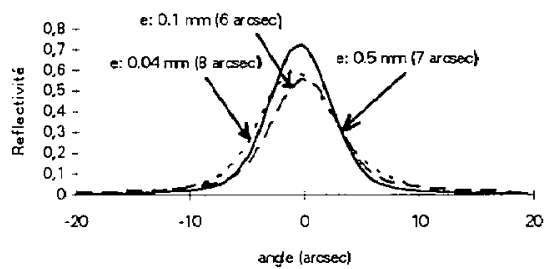

Figure 4: Courbes de diffraction des échantillons silicium/béryllium réalisés avec différentes méthodes d'assemblage (e : épaisseur du silicium)

\section{CONCLUSION}

La simulation thermomécanique et les premiers tests d'assemblage silicium/béryllium ont montré que la réalisation d'un cristal adaptatif composé d'un monocristal de silicium fixé sur un support refroidi en béryllium est théoriquement réalisable et répond au critère des $5 \mu \mathrm{rad}$ que nous nous sommes fixés.

Ce cristal adaptatif refroidi est destiné à compenser les déformations dues au faisceau synchrotron de forte puissance mais un système similaire peut être appliqué à une optique focalisante dont la forme peut être optimisée à application donnée (courbures elliptique, cylindrique ou parabolique). Parallèlement à la réalisation de ce cristal, nous approfondissons la méthode d'assemblage silicium/béryllium afin de s'approcher de l'épaisseur optimale de silicium.

\section{REFERENCES}

1 R. K. Smither, G. A. Forster, D. H. Bilderback, M. Bedzyk, K Finkelstein, C. Henderson, J. White, L. E. Berman, P. Stefan and T. Oversluizen, Rev. Sci. Instrum. 60 (7), (1989) 1486-1492.

2 A. K. Freund, F. de Bergevin, G. Marot, C. Riekel, J. Susini, L. Zhang and E. Ziegler, Opt. Eng. 29 (8), (1990) 928-941.

3 A. K. Freund, Rev. Sci. Instrum. 63 (1), (1992) 413-418.

4 G. Marot, M. Rossat, A. K. Freund, S. Joksch, H. Kawata, L. Zhang, E. Ziegler, L. E. Berman, D. Chapman, J. B. Hastings and M. Iarocci, Rev. Sci. Instrum. 63 (1), (1992) 477-480. 
5 L.E. Berman, M. Hart and S. Sharma, Nucl. Instr. Meth., A 321, (1992) 617-628.

6 J. Susini, G. Förstner, L. Zhang, C. Boyer and R. Ravelet, Rev. Sci. Instrum. 63 (1) (1992), 423-427.

7 J. Susini, G. Marot, L. Zhang, R. Ravelet and P. Jagourel, Rev. Sci. Instrum. 63 (1) (1992), 489-492.

8 D Dézoret, R. Marmoret, A. Freund, R. Ravelet SPIE, San Diego 24-27 July 1994, vol. 2279

9 P. Elleaume, Nucl. Instr. Meth. A 266, (1988) 125-131.

$10 \AA$. Kvick and M. Wulff, Rev. Sci. Instrum. 63 (1) (1992), 1073-1076.

11 A. K. Freund, G. Marot, H. Kawata, S. Joksch, E. Ziegler, L. E. Berman and J. B. Hasting, Rev. Sci. Instrum. 63 (1), (1992) 442-445.

12 D Dézoret, R. Marmoret (cette conférence)

13 J. P. Gaffard, R. Ravelet, C. Boyer, SPIE, San Diego 21-23 July 1992, vol. 1739 , pp.474-488 